\title{
A comparison of different algorithms for phasing haplotypes using Holstein cattle genotypes and pedigree data
}

\author{
Younes Miar, ${ }^{*} \dagger^{1}$ Mehdi Sargolzaei, $† \ddagger$ and Flavio S. Schenkel $\dagger$ \\ *Department of Animal Science and Aquaculture, Dalhousie University, Truro, Nova Scotia, Canada B2N 5E3 \\ †Centre for Genetic Improvement of Livestock, Department of Animal Biosciences, University of Guelph, Guelph, Ontario, Canada N1G 2W1 \\ $\ddagger$ The Semex Alliance, Guelph, Ontario, Canada N1H 6J2
}

\section{ABSTRACT}

Phasing genotypes to haplotypes is becoming increasingly important due to its applications in the study of diseases, population and evolutionary genetics, imputation, and so on. Several studies have focused on the development of computational methods that infer haplotype phase from population genotype data. The aim of this study was to compare phasing algorithms implemented in Beagle, Findhap, FImpute, Impute2, and ShapeIt2 software using 50k and $777 \mathrm{k}$ (HD) genotyping data. Six scenarios were considered: no-parents, sire-progeny pairs, sire-dam-progeny trios, each with and without pedigree information in Holstein cattle. Algorithms were compared with respect to their phasing accuracy and computational efficiency. In the studied population, Beagle and FImpute were more accurate than other phasing algorithms. Across scenarios, phasing accuracies for Beagle and FImpute were 99.49$99.90 \%$ and $99.44-99.99 \%$ for $50 \mathrm{k}$, respectively, and 99.90-99.99\% and $99.87-99.99 \%$ for HD, respectively. Generally, FImpute resulted in higher accuracy when genotypic information of at least one parent was available. In the absence of parental genotypes and pedigree information, Beagle and Impute2 (with double the default number of states) were slightly more accurate than FImpute. Findhap gave high phasing accuracy when parents' genotypes and pedigree information were available. In terms of computing time, Findhap was the fastest algorithm followed by FImpute. FImpute was 30 to 131,87 to 786 , and 353 to 1,400 times faster across scenarios than Beagle, ShapeIt2, and Impute2, respectively. In summary, FImpute and Beagle were the most accurate phasing algorithms. Moreover, the low computational requirement of FImpute makes it an attractive algorithm for phasing genotypes of large livestock populations.

Received June 11, 2016.

Accepted December 9, 2016.

${ }^{1}$ Corresponding author: miar@dal.ca
Key words: haplotype inference, imputation, livestock, phasing accuracy

\section{INTRODUCTION}

Haplotypes are combinations of alleles that are present on each of 2 homologous chromosomes in a diploid individual. Some statistical methods exist for inferring haplotypes from observed genotypes. The inference of a haplotype from genotype data is called "phasing." The importance of haplotype phasing is increasing with the availability of enormous amounts of genotype data generated by high-throughput technologies. Several applications of phasing include imputation of untyped genetic variation (Marchini et al., 2007; Browning and Browning, 2009; Li et al., 2010), interplay of genetic variation and phenotype (Tewhey et al., 2011), population evolutionary history (Tishkoff et al., 1996), linkage disequilibrium mapping, calling genotypes in microarray and sequence data (Kang et al., 2004; Li et al., 2011), detecting genotyping errors (Scheet and Stephens, 2008), inferring points of recombination (Kong et al., 2008), detecting recurrent mutation (Kong et al., 2008), signatures of selection (Sabeti et al., 2002), and modeling cis-regulation of gene expression (Tao et al., 2006). Recently, advances in genotyping technologies and computational approaches have improved the accuracy of haplotyping but experimental methods are expensive and time consuming. On the other hand, computational (in silico) phasing methods are inexpensive but may be time consuming. Computational methods are generally divided into family-based and population-based methods that use linkage information from close relatives and linkage disequilibrium information from population, respectively (Li et al., 2009).

Family-based methods are mostly rule-based methods such as those proposed by Burdick et al. (2006) and Kong et al. (2008). Population-based methods are often based on the stochastic model, and their accuracy depends on sample size, marker density, genotype accuracy, allele frequency, ethnicity, and relatedness (Browning and Browning, 2011). Population-based ap- 
proaches can be highly accurate if high-density markers and large sample sizes are used but they are computationally intensive (Sargolzaei et al., 2014).

The task of phasing in livestock populations is something of a special case, because individuals exhibit much higher levels of relatedness and tend to share much longer stretches of chromosomes compared with individuals in the human population. Lander et al. (1987) used hidden Markov models (HMM) to construct primary genetic linkage maps of experimental and natural populations, which is implemented in Mapmaker. Currently, the most accurate methods use HMM to infer the haplotypes using linkage disequilibrium information (Browning and Browning, 2009; Delaneau et al., 2013; O'Connell et al., 2014). A method proposed by Kong et al. (2008) uses the surrogate parents to infer long haplotypes with high accuracy using Mendelian inheritance rules. Palin et al. (2011) proposed a model based on this approach called "systematic long-range phasing." Meuwissen and Goddard (2010) proposed a combined family and population phasing approach in which family information is used by iterative peeling algorithm following by an approximation of identicalby-descent probabilities. Sargolzaei et al. (2014) developed a rule-based method for phasing that exploited the relationships between individuals based on the fact that close relatives share longer haplotypes and distant relatives share shorter haplotypes.

Because of the relatively small effective population size and planned breeding in most livestock populations, a wide range of structured relationships between individuals is usually observed. For example, large half-sib families are common in livestock. Assessing the performance (i.e., accuracy and computational requirements) of alternative phasing algorithms for livestock populations is important before performing haplotype phasing in research or applied settings. There exist a few investigations on the performance of algorithms for phasing genotypes in livestock, especially using large genotypes and pedigreed data sets. Therefore, the objective of this study was to investigate the phasing accuracy and computing requirements of 5 previously published statistical algorithms for inferring haplotypes from genotype data in a large Holstein cattle population, which were implemented in Beagle (Browning and Browning, 2009), Findhap (VanRaden et al., 2013), FImpute (Sargolzaei et al., 2014), Impute2 with both default and high-accuracy settings (Howie et al., 2009), and ShapeIt2 with both default and high-accuracy settings and the new duoHMM algorithm for scenarios with pedigree information (Delaneau et al., 2013; O'Connell et al., 2014).

\section{MATERIALS AND METHODS}

\section{Data Sets}

To provide a comprehensive assessment of the accuracy of algorithms, 6 different scenarios (3 data subsets with or without pedigree) that vary in the extent of the relatedness between individuals from the North American Holstein genotype database were analyzed. The scenarios are summarized in Table 1 . The data set was provided by the Canadian Dairy Network (CDN, Guelph, ON, Canada), contained 2,495 and 118,946 animals genotyped with Illumina BovineHD (HD) and BovineSNP50 (50k) BeadChips (Illumina Inc., San Diego, CA), respectively. The North American Holstein database contains individuals registered in Canada and the United States. Quality control was performed on $50 \mathrm{k}$ genotypes by the Council on Dairy Cattle Breeding (CDCB, Bowie, MD). Details of quality control measures are given in Wiggans et al. (2009). A total of 45,187 SNP were retained for analysis after filtering. Genotyped animals that were born from 2012 to 2015 with both parents also genotyped comprised the validation set. Genotypes of parents were used to determine phase of heterozygous loci in validation animals, for which parents carried opposing homozygous genotypes. These inferred phases were considered highly accurate and were used to assess haplotype accuracy from different algorithms. The validation set for the 50k panel included 9,266 dairy cattle. Influential genotyped animals, which had more than 40 offspring and were not parents of animals in validation sets, were included in all 6 scenarios for the $50 \mathrm{k}$ panel as influential animals (Table 1). Also, 1,916 animals with HD genotypes that were not parents of validation animals were considered influential animals for the HD panel (Table 1). The percentage of influential animals that were grandparents of validation animals was 0.00 and $1.59 \%$ for the $50 \mathrm{k}$ and HD data sets, respectively. The average pedigree-based relationship between validation and influential animals that had pedigree information were 0.13 (ranged from 0.02 to 0.62 ) and 0.11 (ranged from 0.01 to 0.46 ) for the $50 \mathrm{k}$ and HD data sets, respectively. No-parents scenarios included both the validation animals and the influential animals; the sire-progeny pair scenarios included validation animals, influential animals, and sires; and sire-dam-progeny trio scenarios included validation animals, influential animals, sires, and dams (Table 1).

The validation set for the HD panel included 284 dairy cattle that were genotyped with the HD panel. Parents of validation animals had HD or imputed HD genotypes from $50 \mathrm{k}$ available. Imputation from $50 \mathrm{k}$ 
panel to high density was carried out with FImpute version 2.2 (Sargolzaei et al., 2014) for 197 parents, including 90 sires and 107 dams. The expected imputation accuracy from $50 \mathrm{k}$ panel to $777 \mathrm{k}$ panel by FImpute is about $98.9 \%$ (Sargolzaei et al., 2014). A total of 49 sires and 106 dams were genotyped with the HD panel. The animals with HD genotypes that were not parents of validation animals were also included in all scenarios for the HD panel as influential animals (Table 1). Quality control was applied to HD genotypes data according to VanRaden et al. (2013). A total of 657,932 SNP were retained for the analysis after filtering. In this study, only the SNP on the 29 autosomal bovine chromosomes were considered.

\section{Haplotype Phasing Algorithms}

Haplotypes were reconstructed using various algorithms including FImpute version 2.2 (Sargolzaei et al., 2014), Findhap version 3 (VanRaden et al., 2013), Beagle version 4.1 (Browning and Browning, 2009), ShapeIt2 version 2.12 with default setting (Delaneau et al., 2013), ShapeIt2 version 2.12 with high-accuracy setting, using 200 states (2 times the default setting) and window size of $5.0 \mathrm{Mb}$ (approximately 2.5 times the default setting; Delaneau et al., 2013), ShapeIt2 version 2.12 using new duoHMM algorithm for the scenarios with pedigree information (O'Connell et al., 2014), Impute2 version 2.3.2 with default setting (Howie et al., 2009), and Impute2 version 2.3.2 with high-accuracy setting using 160 states, which is 2 times the states of default setting (Howie et al., 2009).

\section{Comparison of Computational Requirements}

All software were run according to the instructions in their respective manuals. Computation time and maximum resident memory size for phasing chromosome 13 for each software and scenario were measured. Chromosome 13 was chosen because it is the mediansize chromosome among all autosomes in the bovine genome. This chromosome bears 1,519 and 19,091 SNP in the $50 \mathrm{k}$ and HD panels, respectively. All scenarios were run on an Intel Xeon CPU E5-2690 (2.90 GHz) central processing unit (CPU) with $256 \mathrm{~GB}$ of RAM. Beagle 4.1 was run without the "lowmem" option for better performance. Impute2 and ShapeIt2 were run with both default and high-accuracy settings. Although no parallel processing was employed, the option exists for exploiting multiple processors for each software. Although most of the software have the ability to run on $N$ threads, only one thread was employed.

\section{Haplotype Accuracy Based on Switch Error}

The switch error rate (SER) of inferred haplotypes for each scenario was calculated according to Stephens and Donnelly (2003). Switch accuracy is calculated as 1 minus SER. The SER is the number of switches required in the inferred haplotype phase to obtain the

Table 1. Summary of scenarios including the number of offspring, influential animals, sires, dams, pedigree size, and total number of animals used in each scenario

\begin{tabular}{|c|c|c|c|c|c|c|c|}
\hline Scenario $^{1}$ & Chip $^{2}$ & Total & $\begin{array}{c}\text { Offspring } \\
\text { (validation set) }\end{array}$ & Influential $^{3}$ & Sires & Dams & Pedigree \\
\hline \multirow[t]{2}{*}{ No-Parents } & $50 \mathrm{k}$ & 9,670 & 9,266 & 404 & 0 & 0 & No \\
\hline & $\mathrm{HD}$ & 2,200 & 284 & 1,916 & 0 & 0 & No \\
\hline \multirow[t]{2}{*}{ Pairs } & $50 \mathrm{k}$ & 10,123 & 9,266 & 404 & 453 & 0 & No \\
\hline & HD & 2,339 & 284 & 1,916 & 139 & 0 & No \\
\hline \multirow{2}{*}{ Trios } & $50 \mathrm{k}$ & 13,500 & 9,266 & 404 & 453 & 3,377 & No \\
\hline & HD & 2,552 & 284 & 1,916 & 139 & 213 & No \\
\hline \multirow[t]{2}{*}{ No-Parents-Pedigree } & $50 \mathrm{k}$ & 9,670 & 9,266 & 404 & 0 & 0 & Yes \\
\hline & HD & 2,200 & 284 & 1,916 & 0 & 0 & Yes \\
\hline \multirow[t]{2}{*}{ Pairs-Pedigree } & $50 \mathrm{k}$ & 10,123 & 9,266 & 404 & 453 & 0 & Yes \\
\hline & HD & 2,339 & 284 & 1,916 & 139 & 0 & Yes \\
\hline \multirow[t]{2}{*}{ Trios-Pedigree } & $50 \mathrm{k}$ & 13,500 & 9,266 & 404 & 453 & 3,377 & Yes \\
\hline & $\mathrm{HD}$ & 2,552 & 284 & 1,916 & 139 & 213 & Yes \\
\hline
\end{tabular}

${ }^{1}$ No-Parents scenario included both the validation animals and the influential animals; Pairs scenario included validation animals, influential animals and sires; Trios scenario included validation animals, influential animals, sires, and dams; No-Parents-Pedigree scenario included both the validation animals and the influential animals with pedigree information; Pairs-Pedigree scenario included validation animals, influential animals and sires with pedigree information; and Trios-Pedigree scenario included validation animals, influential animals, sires, and dams with pedigree information.

${ }^{2} 50 \mathrm{k}=$ Illumina BovineSNP50 (Illumina Inc., San Diego, CA); HD = Illumina BovineHD.

${ }^{3}$ Animals with more than 40 offspring that were not parents of validation animals were considered influential animals for $50 \mathrm{k}$, and animals with HD genotypes that were not parents of validation animals were also considered for HD panel. 
Table 2. The number of informative heterozygous SNP (possible switches) for each bovine chromosome in the Illumina BovineSNP50 (50k) and BovineHD (HD) BeadChips (Illumina Inc., San Diego, CA)

\begin{tabular}{lcr}
\hline & \multicolumn{2}{c}{ Possible switches } \\
\cline { 2 - 3 } BTA & $50 \mathrm{k}$ & $\mathrm{HD}$ \\
\hline 1 & $6,931,036$ & $2,829,799$ \\
2 & $5,529,291$ & $2,524,854$ \\
3 & $5,365,295$ & $2,296,860$ \\
4 & $5,204,347$ & $2,327,255$ \\
5 & $4,446,385$ & $2,296,747$ \\
6 & $5,111,890$ & $2,399,224$ \\
7 & $4,536,264$ & $2,060,181$ \\
8 & $4,960,879$ & $1,791,892$ \\
9 & $4,196,859$ & $2,080,443$ \\
10 & $4,229,323$ & $2,014,249$ \\
11 & $4,514,040$ & $2,185,098$ \\
12 & $3,475,518$ & $1,741,210$ \\
13 & $3,746,913$ & $1,329,037$ \\
14 & $3,800,447$ & $1,368,576$ \\
15 & $3,570,942$ & $1,693,005$ \\
16 & $3,338,511$ & $1,543,946$ \\
17 & $3,304,677$ & $1,554,231$ \\
18 & $2,925,074$ & $1,414,758$ \\
19 & $2,867,111$ & $1,313,954$ \\
20 & $3,143,393$ & $1,427,177$ \\
21 & $2,901,813$ & $1,373,770$ \\
22 & $2,570,234$ & $1,270,228$ \\
23 & $2,272,421$ & $1,065,292$ \\
24 & $2,512,438$ & $1,252,590$ \\
25 & $2,141,907$ & 938,977 \\
26 & $2,234,355$ & $1,008,199$ \\
27 & $2,070,057$ & 938,271 \\
28 & $2,036,800$ & 961,515 \\
29 & $2,234,343$ & $1,011,318$ \\
\hline
\end{tabular}

true haplotype phase divided by the number of opportunities for SER, which is the number of informative heterozygote markers in the individual's genotype. The number of informative heterozygote markers for the $50 \mathrm{k}$ and HD panels are summarized in Table 2. The size of the validation sets used to evaluate the phasing accuracy were 9,266 and 284 animals for $50 \mathrm{k}$ and HD panels, respectively. All the algorithms produced the whole-chromosome haplotypes for each of the 29 autosomal chromosomes. The effective population size $(\mathrm{Ne})$ for Holstein cattle is approximately 100 (Miar et al., 2015), which was used for all scenarios and algorithms.

\section{RESULTS}

\section{Switch Accuracies}

Haplotype-phasing algorithms were compared using switch accuracy for real 50k genotypes with different sample sizes $(9,670,10,123$, or 13,500$)$ and relatedness (no-parents, sire-progeny pairs, or sire-dam-progeny trios). Table 3 presents the switch accuracies for each haplotype-inference algorithm and scenario, and Figure 1 depicts the switch accuracies for the best-performing algorithms using the 50k panel. Although significant differences for switch accuracies between the algorithms were identified, the actual differences were quite small (between 1 and 3\%). Furthermore, the range of switch accuracies across chromosomes for each algorithm and scenario using 50k panel is presented in Table 4.

Accuracy of haplotype-phasing algorithms was also compared using HD genotypes. Switch accuracies for 3 different sample sizes, each with a different level of relatedness (2,200 of no-parents, 2,339 of sire-progeny pairs, or 2,552 of sire-dam-progeny trios), were evaluated. Table 5 shows switch accuracies for each haplotype-inference algorithm and scenario, and Figure 2 depicts the switch accuracies for the best-performing algorithms using the HD panel. For the HD panel, significant differences in switch accuracies between the algorithms were between 1 and $9 \%$. Furthermore, the range of switch accuracies across chromosomes for each algorithm and scenario using HD panel is presented in Table 6.

Beagle and FImpute consistently gave the best or close to the best switch accuracy. FImpute performed

Table 3. Switch accuracy for various algorithms and scenarios using the Illumina BovineSNP50 BeadChip (Illumina Inc., San Diego, CA)

\begin{tabular}{|c|c|c|c|c|c|c|c|}
\hline Scenario $^{1}$ & FImpute & Findhap & Beagle & $\begin{array}{l}\text { Impute2 } \\
\text { (Default) }\end{array}$ & $\begin{array}{c}\text { Impute2 } \\
\text { (High) }\end{array}$ & $\begin{array}{l}\text { ShapeIt2 } \\
\text { (Default) }\end{array}$ & $\begin{array}{c}\text { ShapeIt2 } \\
\text { (High) }\end{array}$ \\
\hline No-Parents & 99.44 & 97.45 & 99.49 & 98.50 & 99.46 & 98.12 & 98.90 \\
\hline Pairs & 99.86 & 97.43 & 99.67 & 98.43 & 99.46 & 98.02 & 98.85 \\
\hline No-Parents-Pedigree & 99.44 & 97.45 & 99.49 & $98.50^{2}$ & $99.46^{2}$ & 98.13 & 99.51 \\
\hline Pairs-Pedigree & 99.67 & 99.31 & 99.71 & $98.43^{2}$ & $99.46^{2}$ & 98.94 & 99.74 \\
\hline Trios-Pedigree & 99.99 & 99.72 & 99.90 & $98.15^{2}$ & $99.42^{2}$ & 99.11 & 99.80 \\
\hline
\end{tabular}

${ }^{1}$ No-Parents scenario included both the validation animals and the influential animals; Pairs scenario included validation animals, influential animals and sires; Trios scenario included validation animals, influential animals, sires, and dams; No-Parents-Pedigree scenario included both the validation animals and the influential animals with pedigree information; Pairs-Pedigree scenario included validation animals, influential animals and sires with pedigree information; and Trios-Pedigree scenario included validation animals, influential animals, sires, and dams with pedigree information.

${ }^{2}$ Impute2 has no option to input pedigree information. Therefore, the same results are given for scenarios with and without pedigree. 
particularly well with high relatedness, large sample sizes, and high-density genotypes. Increasing the family genotypic information and sample size from no-parents to sire-progeny pairs and from pairs to sire-dam-progeny trios increased switch accuracy for the data sizes considered. The largest improvements were observed from no-parents scenario to pairs scenario for the $50 \mathrm{k}$ data set (Table 3). However, for HD data, the largest improvements were less than those for corresponding $50 \mathrm{k}$ data, because of the increased accuracies due to higher density of markers (Table 5). For the 50k data set, Beagle for no-parents scenario (99.49\%), ShapeIt2 with high-accuracy setting for no-parents-pedigree $(99.51 \%)$ and pairs-pedigree scenarios $(99.74 \%)$, and FImpute for pairs $(99.86 \%)$, trios $(99.98 \%)$, and trios-pedigree scenarios $(99.99 \%)$ outperformed other algorithms (Table 3). However, for HD genotypes, Beagle for no-parents (99.90\%), no-parents-pedigree (99.90\%), pairs-pedigree $(99.95 \%)$, and trios-pedigree scenarios (99.99\%), and FImpute for pairs $(99.94 \%)$, trios $(99.99 \%)$, and triospedigree scenarios $(99.99 \%)$ outperformed other algorithms (Table 5).

For FImpute, the percentage of genotypes whose phase were correctly inferred based on switch accuracy range was $99.44-99.99 \%$ and $99.87-99.99 \%$ across scenarios for 50k and HD, respectively. Beagle also gave the best or close to the best switch accuracies, especially with the HD panel (99.49-99.90\% and 99.90-99.99\%

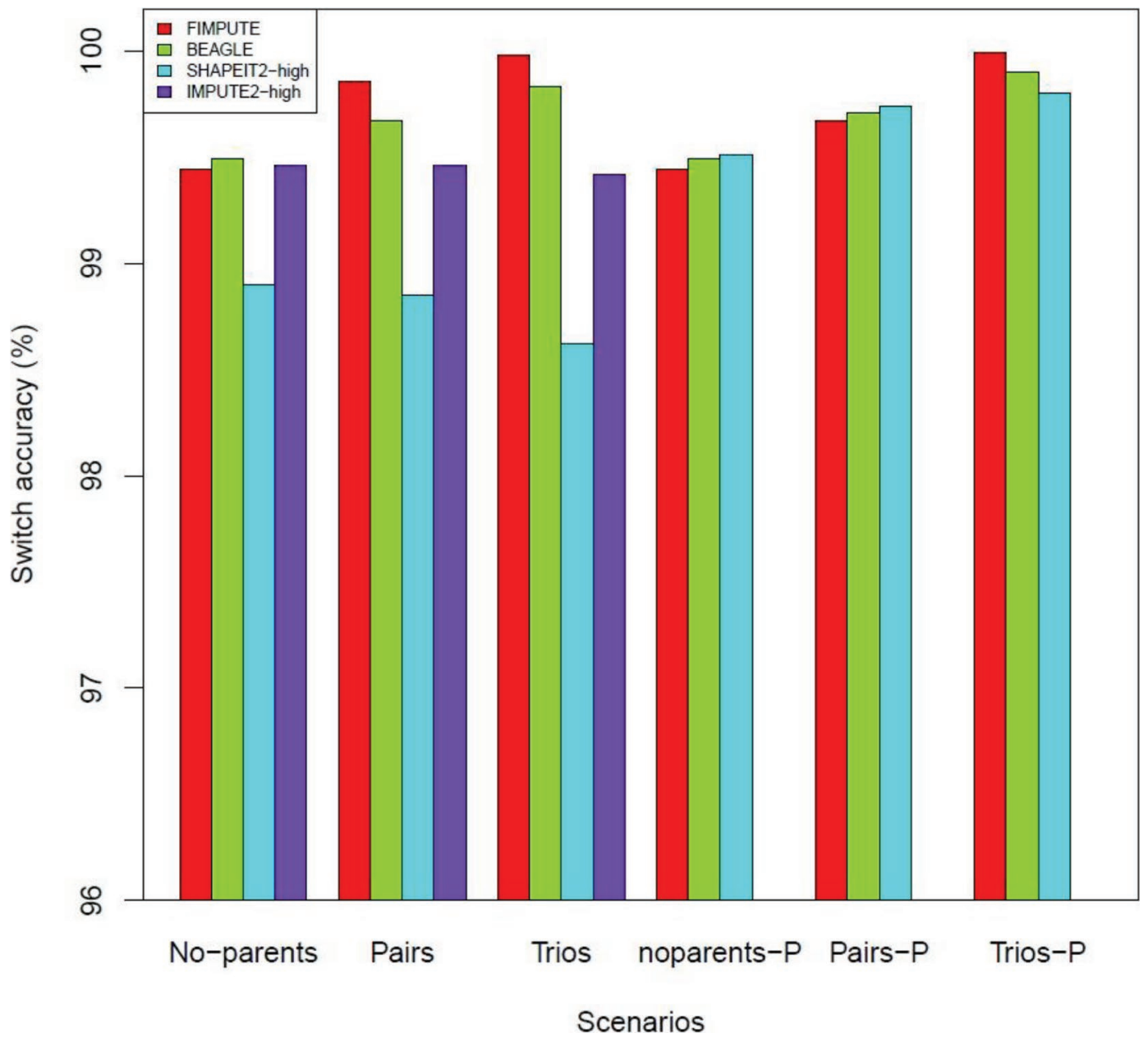

Figure 1. Switch accuracy for best-performing algorithms using Illumina BovineSNP50 BeadChip (Illumina Inc., San Diego, CA). No-parents included both the validation animals and the influential animals; Pairs included validation animals, influential animals and sires; Trios included validation animals, influential animals, sires, and dams; noparents-P included both the validation animals and the influential animals with pedigree information; Pairs-P included validation animals, influential animals and sires with pedigree information; and Trios-P included validation animals, influential animals, sires, and dams with pedigree information. ShapeIt2-high is a high-accuracy setting of ShapeIt2 using 200 states (twice the default) and window size of $5.0 \mathrm{Mb}$, which is approximately 2.5 times larger than default; and Impute2-high is a high-accuracy setting of Impute2 using 160 states, which is twice the states of the default setting. Color version available online. 
Table 4. Range of switch accuracy (BTA number in parentheses) for various algorithms and scenarios using Illumina BovineSNP50 BeadChip (Illumina Inc., San Diego, CA) across Bos taurus autosomes

\begin{tabular}{|c|c|c|c|c|c|c|c|}
\hline Scenario $^{1}$ & FImpute & Findhap & Beagle & $\begin{array}{l}\text { Impute2 } \\
\text { (Default) }\end{array}$ & $\begin{array}{c}\text { Impute2 } \\
\text { (High) }\end{array}$ & $\begin{array}{l}\text { ShapeIt2 } \\
\text { (Default) }\end{array}$ & $\begin{array}{l}\text { ShapeIt2 } \\
\text { (High) }\end{array}$ \\
\hline No-Parents & $\begin{array}{l}99.24(21) \text { to } \\
99.55(4)\end{array}$ & $\begin{array}{l}96.86(22) \text { to } \\
98.15(10)\end{array}$ & $\begin{array}{l}99.12(22) \text { to } \\
99.63(4)\end{array}$ & $\begin{array}{l}95.92(27) \text { to } \\
99.39(1)\end{array}$ & $\begin{array}{l}98.64(26) \text { to } \\
99.70(1)\end{array}$ & $\begin{array}{l}97.25(10) \text { to } \\
98.68(18)\end{array}$ & $\begin{array}{l}98.50(7) \text { to } \\
99.18(15)\end{array}$ \\
\hline Pairs & $\begin{array}{l}99.80(26) \text { to } \\
99.90(1)\end{array}$ & $\begin{array}{l}96.84(25) \text { to } \\
98.12(10)\end{array}$ & $\begin{array}{l}99.53(26) \text { to } \\
99.77(4)\end{array}$ & $\begin{array}{l}95.88(27) \text { to } \\
99.41(1)\end{array}$ & $\begin{array}{l}98.46(26) \text { to } \\
99.71(1)\end{array}$ & $\begin{array}{l}96.88(10) \text { to } \\
98.62(18)\end{array}$ & $\begin{array}{l}98.35(10) \text { to } \\
99.16(15)\end{array}$ \\
\hline Trios & $\begin{array}{l}99.97(5) \text { to } \\
99.98(8)\end{array}$ & $\begin{array}{l}96.94(22) \text { to } \\
98.14(10)\end{array}$ & $\begin{array}{l}99.71(2) \text { to } \\
99.91(4)\end{array}$ & $\begin{array}{l}95.38(27) \text { to } \\
99.47(1)\end{array}$ & $\begin{array}{l}97.74(26) \text { to } \\
99.85(1)\end{array}$ & $\begin{array}{l}96.51 \text { (10) to } \\
98.38(18)\end{array}$ & $\begin{array}{l}98.00(10) \text { to } \\
99.02(18)\end{array}$ \\
\hline Pairs-Pedigree & $\begin{array}{l}99.51(26) \text { to } \\
99.75(9)\end{array}$ & $\begin{array}{l}98.98(6) \text { to } \\
99.49(3)\end{array}$ & $\begin{array}{l}99.57(26) \text { to } \\
99.78(4)\end{array}$ & $\begin{array}{l}95.88(27) \text { to } \\
99.41(1)^{2}\end{array}$ & $\begin{array}{l}98.46(26) \text { to } \\
99.71(1)^{2}\end{array}$ & $\begin{array}{l}98.25(10) \text { to } \\
99.34(18)\end{array}$ & $\begin{array}{l}99.54(6) \text { to } \\
99.82(9)\end{array}$ \\
\hline Trios-Pedigree & $\begin{array}{l}99.99(20) \text { to } \\
99.99(23)\end{array}$ & $\begin{array}{l}99.60(26) \text { to } \\
99.81(15)\end{array}$ & $\begin{array}{l}99.85(26) \text { to } \\
99.94(4)\end{array}$ & $\begin{array}{l}95.38(27) \text { to } \\
99.47(1)^{2}\end{array}$ & $\begin{array}{l}97.74(26) \text { to } \\
99.85(1)^{2}\end{array}$ & $\begin{array}{l}98.55(6) \text { to } \\
99.37(25)\end{array}$ & $\begin{array}{l}99.59(6) \text { to } \\
99.88(1)\end{array}$ \\
\hline
\end{tabular}

${ }^{1}$ No-Parents scenario included both the validation animals and the influential animals; Pairs scenario included validation animals, influential animals and sires; Trios scenario included validation animals, influential animals, sires, and dams; No-Parents-Pedigree scenario included both the validation animals and the influential animals with pedigree information; Pairs-Pedigree scenario included validation animals, influential animals and sires with pedigree information; and Trios-Pedigree scenario included validation animals, influential animals, sires, and dams with pedigree information.

${ }^{2}$ Impute2 has no option to input pedigree information. Therefore, the same results are given for scenarios with and without pedigree.

for 50k and HD, respectively). Compared with other algorithms, Findhap and ShapeIt2 with default setting had lower accuracy, especially for high-density data sets. Findhap switch accuracy range was $97.42-99.72 \%$ for $50 \mathrm{k}$ and $95.85-98.89 \%$ for HD; for ShapeIt2 with default setting, the range was $97.63-99.11 \%$ for $50 \mathrm{k}$ and $91.09-99.73 \%$ for HD. For the data sets considered here, Impute2 and ShapeIt2 with high-accuracy setting always gave more accurate results than their corresponding default settings. Impute 2 with the default setting had switch accuracy range of 98.15-98.50\% and $99.79-99.92 \%$ for $50 \mathrm{k}$ and HD data, respectively. These accuracies were increased to $99.42-99.46 \%$ and 99.86-99.93\% when high-accuracy settings were used for $50 \mathrm{k}$ and HD data, respectively (Tables 3 and 5). ShapeIt2 with the default setting had switch accuracy range of $97.63-99.11 \%$ and $91.09-99.73 \%$ for $50 \mathrm{k}$ and
HD data, respectively. These accuracies were increased to $98.62-99.80 \%$ and $92.21-99.75 \%$ when high-accuracy settings were used for $50 \mathrm{k}$ and HD data, respectively (Tables 3 and 5).

\section{Switch Accuracies Under Different Levels of Relatedness}

For the no-parents scenario, Beagle for both $50 \mathrm{k}$ and HD data sets and Impute2 with high-accuracy setting for 50k data gave slightly higher accuracy than FImpute, but for the scenarios with parental genotypic information, Beagle and Impute2 with high-accuracy yielded lower accuracy than FImpute. Findhap and ShapeIt2 with default setting and Impute2 with default setting had consistently lower switch accuracy than the other phasing algorithms. Their error rates were always

Table 5. Switch accuracy for various algorithms and scenarios using Illumina BovineHD BeadChip (Illumina Inc., San Diego, CA)

\begin{tabular}{|c|c|c|c|c|c|c|c|}
\hline Scenario $^{1}$ & FImpute & Findhap & Beagle & $\begin{array}{l}\text { Impute2 } \\
\text { (Default) }\end{array}$ & $\begin{array}{c}\text { Impute2 } \\
\text { (High) }\end{array}$ & $\begin{array}{l}\text { ShapeIt2 } \\
\text { (Default) }\end{array}$ & $\begin{array}{c}\text { ShapeIt2 } \\
\text { (High) }\end{array}$ \\
\hline No-Parents & 99.88 & 95.85 & 99.90 & 99.79 & 99.86 & 99.68 & 99.71 \\
\hline Pairs & 99.94 & 95.87 & 99.91 & 99.82 & 99.88 & 99.69 & 99.72 \\
\hline No-Parents-Pedigree & 99.87 & 95.85 & 99.90 & $99.79^{2}$ & $99.86^{2}$ & 97.00 & 97.05 \\
\hline Pairs-Pedigree & 99.87 & 98.32 & 99.95 & $99.82^{2}$ & $99.88^{2}$ & 92.18 & 92.21 \\
\hline Trios-Pedigree & 99.99 & 98.89 & 99.99 & $99.92^{2}$ & $99.93^{2}$ & 91.09 & 92.44 \\
\hline
\end{tabular}

${ }^{1}$ No-Parents scenario included both the validation animals and the influential animals; Pairs scenario included validation animals, influential animals and sires; Trios scenario included validation animals, influential animals, sires, and dams; No-Parents-Pedigree scenario included both the validation animals and the influential animals with pedigree information; Pairs-Pedigree scenario included validation animals, influential animals and sires with pedigree information; and Trios-Pedigree scenario included validation animals, influential animals, sires, and dams with pedigree information.

${ }^{2}$ Impute2 has no option to input pedigree information. Therefore, the same results are given for scenarios with and without pedigree. 
at least $70 \%$ higher than those of the best-performing algorithms and often were much higher than that (Tables 3 and 5).

For the most complete scenario of sire-dam-progeny trios-pedigree and 50k genotypes, the algorithms in descending order with regards to switch accuracy were FImpute, Beagle, ShapeIt2 with high-accuracy setting, Findhap, and ShapeIt2 with default setting (Table 3). In addition, the algorithms in descending order with regards to switch accuracy for HD genotypes and triospedigree scenario were FImpute, Beagle, Findhap, ShapeIt2 with high-accuracy setting, and ShapeIt2 with default setting (Table 5).

To assess the presence of possible implicit validation bias, $10 \%$ of validation markers (i.e., heterozygous markers with known phase) were masked for each in- dividual. The masked markers were then imputed with each method and phasing accuracy of masked markers was assessed. The switch accuracies for all the methods and scenarios using both 50k and HD were similar to the previous results. For instance, the switch accuracies for the 50k panel were 99.90, 99.99, 99.73, 99.33, and $98.64 \%$ using trios-pedigree scenario and masked genotypes for Beagle, FImpute, Findhap, Impute2 with high-accuracy setting, and ShapeIt2 with high-accuracy setting algorithms, respectively.

\section{Computational Requirements}

The results for computational time are given in Tables 7 and 8. Generally, computing time increased as the sample size and marker density increased. Findhap and

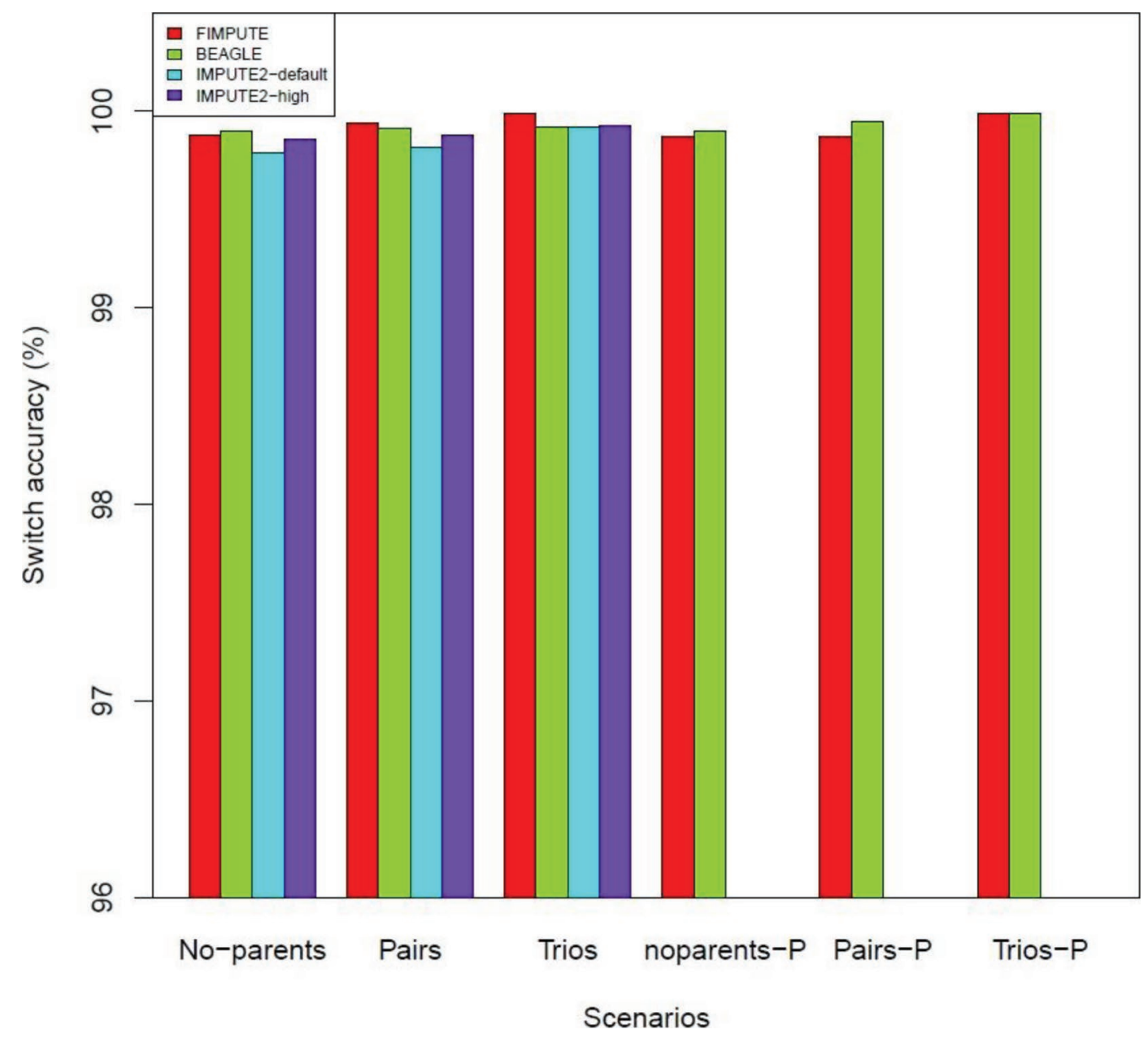

Figure 2. Switch accuracy for best-performing algorithms using Illumina BovineHD BeadChip (Illumina Inc., San Diego, CA). No-parents included both validation animals and influential animals; Pairs included validation animals, influential animals, and sires; Trios included validation animals, influential animals, sires, and dams; noparents-P included both validation animals and influential animals with pedigree information; Pairs-P included validation animals, influential animals, and sires with pedigree information; and Trios-P included validation animals, influential animals, sires, and dams with pedigree information. Impute2-high is a high-accuracy setting of Impute2 using 160 states, which is twice the states of the default setting (Impute2-default). Color version available online. 
Table 6. Range of switch accuracy (BTA number in parentheses) for various algorithms and scenarios using Illumina BovineHD BeadChip (Illumina Inc., San Diego, CA) across Bos taurus autosomes

\begin{tabular}{|c|c|c|c|c|c|c|c|}
\hline Scenario $^{1}$ & FImpute & Findhap & Beagle & $\begin{array}{l}\text { Impute2 } \\
\text { (Default) }\end{array}$ & $\begin{array}{l}\text { Impute2 } \\
\text { (High) }\end{array}$ & $\begin{array}{l}\text { ShapeIt2 } \\
\text { (Default) }\end{array}$ & $\begin{array}{l}\text { ShapeIt2 } \\
\text { (High) }\end{array}$ \\
\hline No-Parents & $\begin{array}{l}99.84(1) \text { to } \\
99.92(24)\end{array}$ & $\begin{array}{l}95.11(27) \text { to } \\
96.71(13)\end{array}$ & $\begin{array}{l}99.85(8) \text { to } \\
99.94(24)\end{array}$ & $\begin{array}{l}99.68(8) \text { to } \\
99.86(18)\end{array}$ & $\begin{array}{l}99.76(8) \text { to } \\
99.90(24)\end{array}$ & $\begin{array}{l}99.57(25) \text { to } \\
99.76(3)\end{array}$ & $\begin{array}{l}99.59 \text { (8) to } \\
99.84(5)\end{array}$ \\
\hline Pairs & $\begin{array}{l}99.92(10) \text { to } \\
99.96(25)\end{array}$ & $\begin{array}{l}95.13(27) \text { to } \\
96.71(13)\end{array}$ & $\begin{array}{l}99.86(8) \text { to } \\
99.94(24)\end{array}$ & $\begin{array}{l}99.74(8) \text { to } \\
99.88(18)\end{array}$ & $\begin{array}{l}99.79(8) \text { to } \\
99.92(24)\end{array}$ & $\begin{array}{l}99.62(8) \text { to } \\
99.78(3)\end{array}$ & $\begin{array}{l}99.61(8) \text { to } \\
99.83(5)\end{array}$ \\
\hline Trios & $\begin{array}{l}99.98(22) \text { to } \\
99.99(26)\end{array}$ & $\begin{array}{l}95.15(27) \text { to } \\
96.73(13)\end{array}$ & $\begin{array}{l}99.90(21) \text { to } \\
99.95(24)\end{array}$ & $\begin{array}{l}99.83(23) \text { to } \\
99.95(25)\end{array}$ & $\begin{array}{l}99.91(21) \text { to } \\
99.96(24)\end{array}$ & $\begin{array}{l}99.65(8) \text { to } \\
99.80(3)\end{array}$ & $\begin{array}{l}99.64(8) \text { to } \\
99.83(17)\end{array}$ \\
\hline Pairs-Pedigree & $\begin{array}{l}99.82(1) \text { to } \\
99.92(25)\end{array}$ & $\begin{array}{l}98.00(8) \text { to } \\
98.71(23)\end{array}$ & $\begin{array}{l}99.94(1) \text { to } \\
99.97(24)\end{array}$ & $\begin{array}{l}99.74(8) \text { to } \\
99.88(18)^{2}\end{array}$ & $\begin{array}{l}99.79(8) \text { to } \\
99.92(24)^{2}\end{array}$ & $\begin{array}{l}90.12(23) \text { to } \\
93.53(16)\end{array}$ & $\begin{array}{l}90.12(23) \text { to } \\
93.55(16)\end{array}$ \\
\hline Trios-Pedigree & $\begin{array}{l}99.99(21) \text { to } \\
99.99(13)\end{array}$ & $\begin{array}{l}98.67 \text { (8) to } \\
99.20(23)\end{array}$ & $\begin{array}{l}99.98(21) \text { to } \\
99.99(24)\end{array}$ & $\begin{array}{l}99.83(23) \text { to } \\
99.95(25)^{2}\end{array}$ & $\begin{array}{l}99.91(21) \text { to } \\
99.96(24)^{2}\end{array}$ & $\begin{array}{l}88.62(27) \text { to } \\
92.59(7)\end{array}$ & $\begin{array}{l}90.19(23) \text { to } \\
93.35(16)\end{array}$ \\
\hline
\end{tabular}

${ }^{1}$ No-Parents scenario included both the validation animals and the influential animals; Pairs scenario included validation animals, influential animals and sires; Trios scenario included validation animals, influential animals, sires, and dams; No-Parents-Pedigree scenario included both the validation animals and the influential animals with pedigree information; Pairs-Pedigree scenario included validation animals, influential animals and sires with pedigree information; and Trios-Pedigree scenario included validation animals, influential animals, sires, and dams with pedigree information.

${ }^{2}$ Impute2 has no option to input pedigree information. Therefore, the same results are given for scenarios with and without pedigree.

FImpute were, by far, the fastest algorithms. Beagle was the next fastest, followed in order by ShapeIt2 with high-accuracy setting, Impute2 with default setting, ShapeIt2 with default setting, and Impute2 with highaccuracy setting (Tables 7 and 8). Figures 3 and 4 depict computation time for the fastest algorithms - Findhap, FImpute, and Beagle - using 50k and HD data sets, respectively. The differences in processing time among algorithms are large enough to have practical significance, especially between fastest algorithms (Findhap and FImpute) and the other investigated algorithms.

Although Findhap was the fastest algorithm, it yielded low phasing accuracy for scenarios without pedigree and no-parents-pedigree, but its phasing accuracy improved substantially when parents' genotypes and pedigree information were used (Tables 3 and 5). It is noteworthy that pedigree and parents' genotypes are mostly available in dairy cattle and Findhap performed well in this scenario.

When comparing FImpute to Beagle, ShapeIt2 with high-accuracy setting, Impute2 with default setting, ShapeIt2 with default setting, and Impute2 with highaccuracy setting, there were 47 - to 131 -fold, 178 - to 345 -fold, 428- to 657-fold, 377- to 786-fold, and 727to 1,007 -fold differences in timing results for the $50 \mathrm{k}$ data set, respectively (Table 7 ). For the HD data set, FImpute was 30 to 40,150 to 270,353 to 390,87 to 158 , and 866 to 931 times faster than Beagle, ShapeIt2 with high-accuracy setting, Impute2 with default setting, ShapeIt2 with default setting, and Impute2 with high-accuracy setting, respectively (Table 8). Depending on the data set, FImpute was 4 to 6 times slower than Findhap, which could be important for phasing the whole North American Holstein database contain-

Table 7. Computing time (hours:minutes:seconds) for 50k genotypes for various algorithms and scenarios

\begin{tabular}{|c|c|c|c|c|c|c|c|}
\hline Scenario $^{1}$ & FImpute & Findhap & Beagle & $\begin{array}{l}\text { Impute2 } \\
\text { (Default) }\end{array}$ & $\begin{array}{c}\text { Impute2 } \\
\text { (High) }\end{array}$ & $\begin{array}{l}\text { ShapeIt2 } \\
\text { (Default) }\end{array}$ & $\begin{array}{c}\text { ShapeIt2 } \\
\text { (High) }\end{array}$ \\
\hline No-Parents & $00: 01: 52$ & 00:00:19 & $01: 26: 45$ & $13: 19: 29$ & $22: 38: 02$ & $16: 11: 47$ & $08: 27: 04$ \\
\hline No-Parents-Pedigree & $00: 01: 53$ & 00:00:19 & $01: 29: 27$ & $13: 19: 29^{2}$ & $22: 38: 02$ & $11: 51: 19$ & $05: 48: 51$ \\
\hline Pairs-Pedigree & 00:02:01 & 00:00:26 & 02:06:40 & 14:50:13 & 44:03:54 & $13: 29: 13$ & $05: 59: 17$ \\
\hline Trios-Pedigree & 00:03:01 & 00:00:30 & 04:59:02 & $23: 43: 53$ & $36: 22: 31$ & $24: 06: 54$ & 09:25:39 \\
\hline
\end{tabular}

${ }^{1}$ No-Parents scenario included both the validation animals and the influential animals; Pairs scenario included validation animals, influential animals and sires; Trios scenario included validation animals, influential animals, sires, and dams; No-Parents-Pedigree scenario included both the validation animals and the influential animals with pedigree information; Pairs-Pedigree scenario included validation animals, influential animals and sires with pedigree information; and Trios-Pedigree scenario included validation animals, influential animals, sires, and dams with pedigree information.

${ }^{2}$ Impute2 has no option to input pedigree information. Therefore, the same results are given for scenarios with and without pedigree. 
Table 8. Computing time (hours:minutes:seconds) for high-density (HD) genotypes for various algorithms and scenarios

\begin{tabular}{|c|c|c|c|c|c|c|c|}
\hline Scenario $^{1}$ & FImpute & Findhap & Beagle & $\begin{array}{l}\text { Impute2 } \\
\text { (Default) }\end{array}$ & $\begin{array}{c}\text { Impute2 } \\
\text { (High) }\end{array}$ & $\begin{array}{l}\text { ShapeIt2 } \\
\text { (Default) }\end{array}$ & $\begin{array}{c}\text { ShapeIt2 } \\
\text { (High) }\end{array}$ \\
\hline No-Parents & 00:02:39 & 00:00:40 & $01: 26: 55$ & $16: 15: 56$ & $40: 57: 28$ & $04: 10: 52$ & $11: 56: 50$ \\
\hline Pairs & 00:03:03 & 00:00:46 & $01: 36: 30$ & $17: 57: 07$ & $44: 03: 54$ & 04:26:20 & $12: 32: 21$ \\
\hline No-Parents-Pedigree & 00:02:47 & 00:00:40 & 01:35:58 & $16: 15: 56^{2}$ & $40: 57: 28$ & $04: 36: 48$ & $07: 12: 26$ \\
\hline Pairs-Pedigree & 00:03:06 & 00:00:49 & $01: 58: 54$ & $17: 57: 07$ & $44: 03: 54$ & 05:06:54 & $07: 44: 08$ \\
\hline Trios-Pedigree & 00:03:19 & 00:00:54 & $02: 12: 50$ & $21: 08: 58$ & $50: 26: 36$ & 05:57:02 & 09:11:10 \\
\hline
\end{tabular}

${ }^{1}$ No-Parents scenario included both the validation animals and the influential animals; Pairs scenario included validation animals, influential animals and sires; Trios scenario included validation animals, influential animals, sires, and dams; No-Parents-Pedigree scenario included both the validation animals and the influential animals with pedigree information; Pairs-Pedigree scenario included validation animals, influential animals and sires with pedigree information; and Trios-Pedigree scenario included validation animals, influential animals, sires, and dams with pedigree information.

${ }^{2}$ Impute2 has no option to input pedigree information. Therefore, the same results are given for scenarios with and without pedigree.

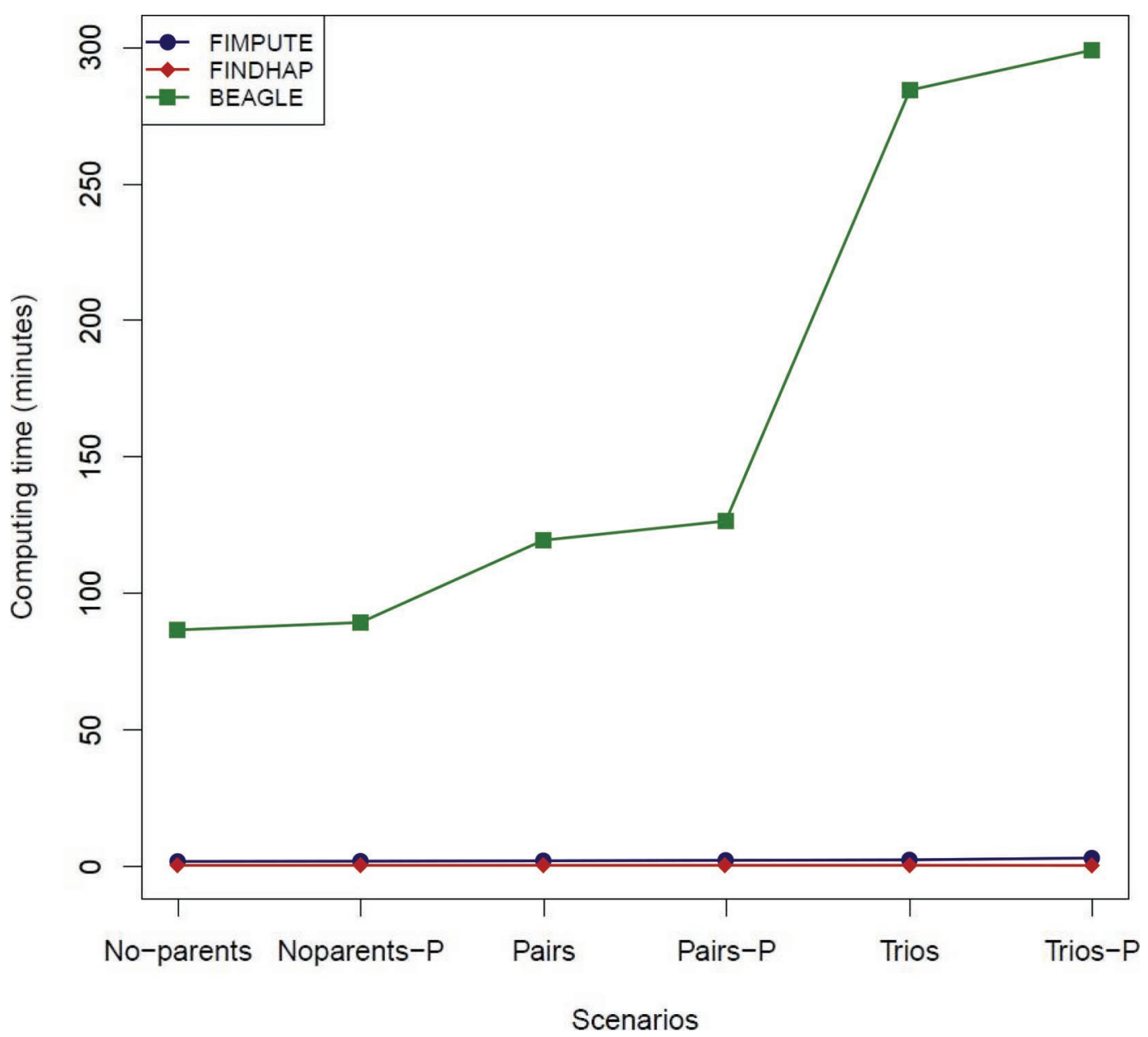

Figure 3. Computing time for the fastest algorithms using Illumina BovineSNP50 BeadChip (Illumina Inc., San Diego, CA). No-parents included both validation animals and influential animals; Noparents-P included both validation animals and influential animals with pedigree information; Pairs included validation animals, influential animals, and sires; Pairs-P included validation animals, influential animals, and sires with pedigree information; Trios included validation animals, influential animals, sires, and dams; and Trios-P included validation animals, influential animals, sires, and dams with pedigree information. Color version available online. 


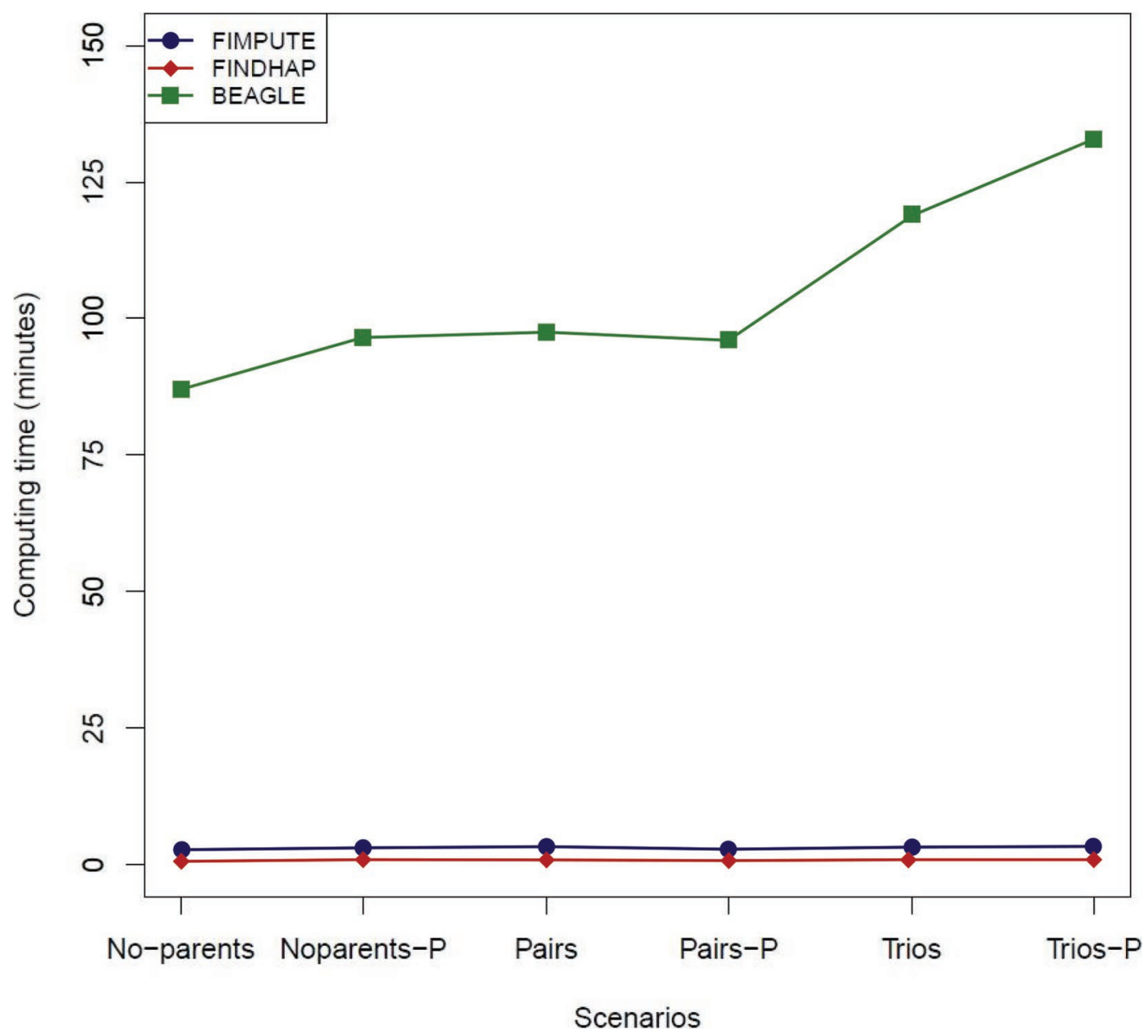

Figure 4. Computing time for the fastest algorithms using Illumina BovineHD BeadChip (Illumina Inc., San Diego, CA). No-parents included both validation animals and influential animals; Noparents-P included both validation animals and influential animals with pedigree information; Pairs included validation animals, influential animals, and sires; Pairs-P included validation animals, influential animals, and sires with pedigree information; Trios included validation animals, influential animals, sires, and dams; and Trios-P included validation animals, influential animals, sires, and dams with pedigree information. Color version available online.

ing 2,495 and 118,946 animals genotyped with Illumina BovineHD and BovineSNP50 BeadChips, respectively. Other methods; that is, Beagle, Impute2, and ShapeIt2, cannot handle such a large data set. For FImpute, Findhap, and Impute2, computational time increased as marker density increased, but Beagle and ShapeIt2 phased HD faster than 50k.

Based on the memory usage given in Tables 9 and 10, the following patterns were observed: FImpute required less memory compared with the other algorithms. Findhap was next, followed by ShapeIt2, Impute2 with default setting, Impute2 with high-accuracy setting, and Beagle. For the 50k data set, Findhap, ShapeIt2, Impute2 with default setting, Impute2 with high-accuracy setting, and Beagle used 2, 7 to 8,47 to 56,49 to 58, and 148 to 194 times more memory than FImpute (Table 9). When phasing HD genotypes, Findhap, ShapeIt2, Impute2 with default setting, Impute2 with high-accuracy setting, and Beagle used 5 to 7,4 to 7 , 36 to 40,41 to 44 , and 32 to 53 times more memory than FImpute (Table 10). These differences in memory usage are significant for routine genomic analyses of large data set.

Computation time and memory usage were compared only for chromosome 13 with a range of 9,670 to 13,500 animals for 50k data set and 2,200 to 2,552 animals for HD data set. For phasing the whole genome, the running time would be much more for the less computationally efficient algorithms, such as Impute2, ShapeIt2, and Beagle; however, Findhap could handle these data sets in a few minutes and FImpute in 10 to $20 \mathrm{~min}$.

\section{DISCUSSION}

The accuracy of phasing depends mainly on the number of individuals, marker density, and relatedness 
Table 9. Maximum memory usage for 50k genotypes (in megabytes) for various algorithms and scenarios

\begin{tabular}{|c|c|c|c|c|c|c|c|}
\hline Scenario $^{1}$ & FImpute & Findhap & Beagle & $\begin{array}{l}\text { Impute2 } \\
\text { (Default) }\end{array}$ & $\begin{array}{c}\text { Impute2 } \\
\text { (High) }\end{array}$ & $\begin{array}{l}\text { ShapeIt2 } \\
\text { (Default) }\end{array}$ & $\begin{array}{c}\text { ShapeIt2 } \\
\text { (High) }\end{array}$ \\
\hline No-Parents & 78 & 171 & 11,929 & 3,691 & 3,807 & 529 & 529 \\
\hline Pairs & 79 & 171 & 12,696 & 3,861 & 3,977 & 554 & 554 \\
\hline No-Parents-Pedigree & 74 & 171 & 12,195 & $3,691^{2}$ & 3,807 & 530 & 530 \\
\hline Pairs-Pedigree & 75 & 171 & 11,941 & 3,861 & 3,977 & 555 & 555 \\
\hline Trios-Pedigree & 102 & 174 & 19,820 & 5,133 & 5,249 & 742 & 742 \\
\hline
\end{tabular}

${ }^{1}$ No-Parents scenario included both the validation animals and the influential animals; Pairs scenario included validation animals, influential animals and sires; Trios scenario included validation animals, influential animals, sires, and dams; No-Parents-Pedigree scenario included both the validation animals and the influential animals with pedigree information; Pairs-Pedigree scenario included validation animals, influential animals and sires with pedigree information; and Trios-Pedigree scenario included validation animals, influential animals, sires, and dams with pedigree information.

${ }^{2}$ Impute2 has no option to input pedigree information. Therefore, the same results are given for scenarios with and without pedigree.

(Browning and Browning, 2011). The most accurate algorithms were FImpute, Beagle, ShapeIt2 with highaccuracy setting, and Impute2 with high-accuracy setting across scenarios. Computational time and ability to analyze large data sets are paramount because different algorithms inferred haplotypes with close phasing accuracies. Although multithreading could reduce computational time, only one thread was used to enable comparison between different algorithms. FImpute was the most accurate phasing algorithm and was capable of phasing all chromosomes with low computational demands. The phasing algorithm implemented in FImpute is deterministic and hence it is computationally more efficient than the HMM used by Beagle and Impute2 software (Browning and Browning, 2009; Howie et al., 2009). Computational time results suggest that the best competing algorithms would require days or weeks of computing time to complete the phasing and, in many instances, with less accuracy. In addition, results show that the best competing algorithm would use $\sim 2$ to 200 times more memory to phase only one chromosome compared with FImpute. FImpute took 10 to $15 \mathrm{~min}$ and 75 to $102 \mathrm{MB}$ of memory to phase $50 \mathrm{k}$ genotypes for 9,670 to 13,500 individuals. Based on the results presented in Tables 3 and 7, the switch accuracy achieved by FImpute was, on average, $99.73 \%$ for $50 \mathrm{k}$ and $99.92 \%$ for HD data sets depending on the parental genotypic information available. Because FImpute was used for imputation of some animals from 50k to HD, lower switch accuracy might be attributed to imputation errors. However, the effect of imputation error is expected to be minimal because of the high accuracy of imputation from 50k to HD using FImpute. For the most complete scenario of sire-dam-progeny trios with pedigree information, FImpute showed high phasing accuracy and low computational requirements. It is expected that the phasing of Illumina BovineSNP50 BeadChip for approximately 13,500 animals with FImpute would take approximately 10 to $15 \mathrm{~min}$ (Table 7), with approximately $99.99 \%$ switch accuracy (Table 3). Similarly, we would expect the phasing of Illumina BovineHD for approximately 2,500 animals with FImpute to take 15 to $20 \mathrm{~min}$ (Table 8), with approximately 99.99\% switch accuracy (Table 5). The switch accuracy achieved here with some algorithms might have been affected by genotyping error, although strict filtering was performed. The genotypic information of the sire and dam or family information is critical for lower

Table 10. Maximum memory usage for high-density (HD) genotypes (in megabytes) for various algorithms and scenarios

\begin{tabular}{|c|c|c|c|c|c|c|c|}
\hline Scenario $^{1}$ & FImpute & Findhap & Beagle & $\begin{array}{l}\text { Impute2 } \\
\text { (Default) }\end{array}$ & $\begin{array}{c}\text { Impute2 } \\
\text { (High) }\end{array}$ & $\begin{array}{l}\text { ShapeIt2 } \\
\text { (Default) }\end{array}$ & $\begin{array}{c}\text { ShapeIt2 } \\
\text { (High) }\end{array}$ \\
\hline No-Parents & 306 & 1,991 & 16,050 & 11,003 & 12,459 & 1,063 & 1,064 \\
\hline Pairs & 313 & 1,993 & 10,099 & 11,660 & 13,117 & 1,125 & 1,126 \\
\hline No-Parents-Pedigree & 348 & 1,991 & 10,681 & $11,003^{2}$ & 12,459 & 1,337 & 1,337 \\
\hline Pairs-Pedigree & 356 & 1.993 & 13,891 & 11,660 & 13,117 & 1,769 & 1,769 \\
\hline Trios-Pedigree & 369 & 1,995 & 13,290 & 12,669 & 14,125 & 2,446 & 2,446 \\
\hline
\end{tabular}

${ }^{1}$ No-Parents scenario included both the validation animals and the influential animals; Pairs scenario included validation animals, influential animals and sires; Trios scenario included validation animals, influential animals, sires, and dams; No-Parents-Pedigree scenario included both the validation animals and the influential animals with pedigree information; Pairs-Pedigree scenario included validation animals, influential animals and sires with pedigree information; and Trios-Pedigree scenario included validation animals, influential animals, sires, and dams with pedigree information.

${ }^{2}$ Impute2 has no option to input pedigree information. Therefore, the same results are given for scenarios with and without pedigree. 
density phasing. However, family information becomes less important as marker density increases. Therefore, the largest improvements of phasing accuracy were achieved when information of at least one parent was added to the analysis for the 50k data set. As expected, the phasing accuracy from denser SNP panels was higher, resulting in less dependency on the availability of close family information. In comparing phasing algorithms, lesser differences in the switch accuracy for the HD data set were observed compared with the 50k data set. The results were consistent across the different levels of relatedness. Although the switch accuracies for Findhap and ShapeIt2 with the new duoHMM algorithm were reduced when high-density markers were phased. The combination of novel duoHMM algorithm and ShapeIt2 was designed to phase any cohort with any levels of implicit or explicit relatedness between individuals (O'Connell et al., 2014). This algorithm incorporates the pedigree information in a cohort aiming to further increase the accuracy of haplotype phasing, although our results showed that phasing accuracy was reduced when duoHMM was added in the scenarios with pedigree information. Precisely determining why this reduction happened is difficult. Using a higher effective population size of 1,000 for the trios-pedigree scenario gave the same switch accuracy as an effective population size of 100, when tested for chromosome 13. Therefore, it seems the low effective population size might not explain the observed reduction in accuracy of haplotyping phasing in this study when duoHMM was added in the scenarios with pedigree information. Assuming an effective population size of 100, Impute2 performed well in the genotype imputation of Swedish and Finnish Red cattle (Ma et al., 2013), further indicating that a low effective population size does not substantially influence the accuracy of iterative algorithms such as ShapeIt2 and Impute2.

Impute 2 performance was better for HD than for $50 \mathrm{k}$ data; however, the computational requirements were very high. Phasing of all chromosomes with Impute2, especially with the high-accuracy setting, for the HD data set would take around 3 wk (Table 10). However, Impute2 was consistently less accurate than Beagle and FImpute, especially for scenarios with parental genotypic information. Impute 2 does not have any option for using pedigree information and, hence, only 3 scenarios without pedigree were compared.

The algorithm implemented in FImpute (Sargolzaei et al., 2014) first uses the pedigree information via an iterative approach for accurate phasing. In the first step, an overlapping sliding window approach is exploited to capture long haplotype information from close relatives by moving long windows over a chromo- some. Thereafter, more distant relatives are assessed by making the window size progressively shorter in each chromosomal sweep. A haplotype library is built for each window that is used for haplotype reconstruction.

Long-range phasing has great potential for genomic analysis, especially as relatedness increases and, hence, more identity-by-descent is shared between animals. Similar to the long-range phasing algorithm developed by Kong et al. (2008), the algorithm implemented in FImpute identifies long shared chromosomal segments. However, FImpute is neither iterative nor uses surrogate parents. In this study, a data set containing substantial level of relatedness among a large Holstein cattle population was used, allowing the long-range phasing approach to infer haplotypes more accurately.

FImpute was the most accurate algorithm capable of phasing all chromosomes with relatively low computational requirements. FImpute owes its accuracy and computational efficiency to the overlapping sliding window approach. It utilizes information from close to distant relatives, resulting in accurate haplotype inferences. The other algorithms-Beagle, Impute2, and ShapeIt2 - presumably could be improved for large data sets by adjusting the default parameters, as we did for Impute2 and ShapeIt2. However, adjustments in default parameters would infer more accurate haplotypes but at the cost of longer computing times and more memory usage. FImpute yielded the most consistent range of switch accuracies across chromosomes for each scenario, which is important for applications such as whole-genome association analysis (Tables 4 and 6).

\section{ACKNOWLEDGMENTS}

The authors acknowledge the Canadian Dairy Network (CDN, Guelph, ON, Canada) for providing the North American Holstein genotype database used in this study. This work was financially supported by Canadian Dairy Research Cluster II grant (Dairy Farmers of Canada, Ottawa, and Agriculture and Agri-Food Canada, Ottawa), which is gratefully acknowledged.

\section{REFERENCES}

Browning, B. L., and S. R. Browning. 2009. A unified approach to genotype imputation and haplotype-phase inference for large datasets of trios and unrelated individuals. Am. J. Hum. Genet. $84: 210-223$

Browning, S. R., and B. L. Browning. 2011. Haplotype phasing: Existing methods and new developments. Nat. Rev. Genet. 12:703-714.

Burdick, J. T., W. M. Chen, G. R. Abecasis, and V. G. Cheung. 2006. In silico method for inferring genotypes in pedigrees. Nat. Genet. 38:1002-1004.

Delaneau, O., J. F. Zagury, and J. Marchini. 2013. Improved whole chromosome phasing for disease and population genetic studies. Nat. Methods 10:5-6. 
Howie, B. N., P. Donnelly, and J. Marchini. 2009. A flexible and accurate genotype imputation method for the next generation of genome-wide association studies. PLoS Genet. 5:e1000529.

Kang, H., Z. S. Qin, T. Niu, and J. S. Liu. 2004. Incorporating genotyping uncertainty in haplotype inference for single-nucleotide polymorphisms. Am. J. Hum. Genet. 74:495-510.

Kong, A., G. Masson, M. L. Frigge, A. Gylfason, P. Zusmanovich, G. Thorleifsson, P. I. Olason, A. Ingason, S. Steinberg, T. Rafnar, P. Sulem, M. Mouy, F. Jonsson, U. Thorsteinsdottir, D. F. Gudbjartsson, H. Stefansson, and K. Stefansson. 2008. Detection of sharing by descent, long-range phasing and haplotype imputation. Nat. Genet. 40:1068-1075.

Lander, E. S., P. Green, J. Abrahamson, A. Barlow, M. Daly, S. Lincoln, and L. A. Newberg. 1987. MAPMAKER: An interactive computer package for constructing primary genetic linkage maps of experimental and natural populations. Genomics 1:174-181.

Li, Y., C. Sidore, H. M. Kang, M. Boehnke, and G. R. Abecasis. 2011. Low-coverage sequencing: Implications for design of complex trait association studies. Genome Res. 21:940-951.

Li, Y., C. J. Willer, J. Ding, P. Scheet, and G. R. Abecasis. 2010. $\mathrm{MaCH}$ : Using sequence and genotype data to estimate haplotypes and unobserved genotypes. Genet. Epidemiol. 34:816-834.

Li, Y., C. J. Willer, S. Sanna, and G. R. Abecasis. 2009. Genotype imputation. Annu. Rev. Genomics Hum. Genet. 10:387-406.

Ma, P., R. F. Brøndum, Q. Zhang, M. S. Lund, and G. Su. 2013. Comparison of different methods for imputing genome-wide marker genotypes in Swedish and Finnish Red cattle. J. Dairy Sci. 96:4666-4677.

Marchini, J., B. Howie, S. Myers, G. McVean, and P. Donnelly. 2007. A new multipoint method for genome-wide association studies by imputation of genotypes. Nat. Genet. 39:906-913.

Meuwissen, T., and M. E. Goddard. 2010. The use of family relationships and linkage disequilibrium to impute phase and missing genotypes in up to whole genome sequence density genotypic data. Genetics 185:1441-1449.

Miar, Y., G. S. Plastow, and Z. Wang. 2015. Genomic selection, a new era for improvement of meat quality. Springer Sci. Rev. 3:27-37.

O'Connell, J., D. Gurdasani, O. Delaneau, N. Pirastu, S. Ulivi, M. Cocca, M. Traglia, J. Huang, J. E. Huffman, I. Rudan, R. McQuillan, R. M. Fraser, H. Campbell, O. Polasek, G. Asiki, K. Ekoru,
C. Hayward, A. F. Wright, V. Vitart, P. Navarro, J. F. Zagury, J. F. Wilson, D. Toniolo, P. Gasparini, N. Soranzo, M. S. Sandhu, and J. Marchini. 2014. A general approach for haplotype phasing across the full spectrum of relatedness. PLoS Genet. 10:e1004234.

Palin, K., H. Campbell, A. F. Wright, J. F. Wilson, and R. Durbin. 2011. Identity-bydescent-based phasing and imputation in founder populations using graphical models. Genet. Epidemiol. 35:853-860.

Sabeti, P. C., D. E. Reich, J. M. Higgins, H. Z. P. Levine, D. J. Richter, S. F. Schaffner, S. B. Gabriel, J. V. Platko, N. J. Patterson, G. J. McDonald, H. C. Ackerman, S. J. Campbell, D. Altshuler, R. Cooper, D. Kwiatkowski, R. Ward, and E. S. Lander. 2002. Detecting recent positive selection in the human genome from haplotype structure. Nature 419:832-837.

Sargolzaei, M., J. P. Chesnais, and F. S. Schenkel. 2014. A new approach for efficient genotype imputation using information from relatives. BMC Genomics 15:478.

Scheet, P., and M. Stephens. 2008. Linkage disequilibrium-based quality control for large-scale genetic studies. PLoS Genet. 4:e1000147.

Stephens, M., and P. Donnelly. 2003. A comparison of Bayesian methods for haplotype reconstruction from population genotype data. Am. J. Hum. Genet. 73:1162-1169.

Tao, H., D. R. Cox, and K. A. Frazer. 2006. Allele-specific KRT1 expression is a complex trait. PLoS Genet. 2:e93.

Tewhey, R., V. Bansal, A. Torkamani, E. J. Topol, and N. J. Schork. 2011. The importance of phase information for human genomics. Nat. Rev. Genet. 12:215-223.

Tishkoff, S. A., E. Dietzsch, W. Speed, A. J. Pakstis, J. R. Kidd, K. Cheung, B. Bonné-Tamir, A. S. Santachiara-Benerecetti, P. Moral, and M. Krings. 1996. Global patterns of linkage disequilibrium at the CD4 locus and modern human origins. Science 271:1380-1387.

VanRaden, P. M., D. J. Null, M. Sargolzaei, G. R. Wiggans, M. E. Tooker, J. B. Cole, T. S. Sonstegard, E. E. Connor, M. Winters, and J. B. van Kaam. 2013. Genomic imputation and evaluation using high density Holstein genotypes. J. Dairy Sci. 96:668-678.

Wiggans, G. R., T. S. Sonstegard, P. M. VanRaden, L. K. Matukumalli, R. D. Schnabel, J. F. Taylor, F. S. Schenkel, and C. P. Van Tassell. 2009. Selection of single-nucleotide polymorphisms and quality of genotypes used in genomic evaluation of dairy cattle in the United States and Canada. J. Dairy Sci. 92:3431-3436. 